\title{
Objective assessment of motor fatigue in multiple sclerosis: the Fatigue index Kliniken Schmieder (FKS)
}

\author{
Aida Sehle • Manfred Vieten - Simon Sailer • \\ Annegret Mündermann · Christian Dettmers
}

\begin{abstract}
Fatigue is a common and frequently disabling symptom of multiple sclerosis (MS). The aim of this study was to develop the Fatigue index Kliniken Schmieder (FKS) for detecting motor fatigue in patients with MS using kinematic gait analysis. The FKS relies on the chaos theoretical term "attractor", which, if unchanged, is a necessary and sufficient indicator of a stable dynamical system. We measured the acceleration of the feet at the beginning of and shortly before stopping a treadmill walking task in 20 healthy subjects and 40 patients with multiple sclerosis. The attractor and movement variability were calculated. In the absence of muscular exhaustion a significant difference in the attractor and movement variability between the two time points demonstrates altered motor control indicating fatigue. Subjects were classified using the FKS. All healthy subjects had normal FKS and thus no fatigue. 29 patients with MS were classified into a fatigue group and 11 patients into a non-fatigue group. This
\end{abstract}

A. Sehle $\cdot$ M. Vieten $\cdot$ A. Mündermann

Division of Sport Science, University of Konstanz,

78457 Constance, Germany

\section{A. Sehle $(\square)$}

Kliniken Schmieder Allensbach, Zum Tafelholz 8,

78476 Allensbach, Germany

e-mail: aida.sehle@uni-konstanz.de

S. Sailer · C. Dettmers

Kliniken Schmieder Konstanz, 78464 Constance, Germany

A. Mündermann

School of Physiotherapy, University of Otago, 9016 Dunedin,

New Zealand

A. Mündermann

Department of Orthopaedics, University Hospital Basel,

4031 Basel, Switzerland classification agreed with the physician's observation and video analyses in up to $97 \%$ of cases. The FKS did not correlate significantly with the overall and motor dimensions of the fatigue questionnaire scores in patients with MS and motor fatigue. The common concept of fatigue as overall subjective sensation of exhaustion can be affected by conditions including depression, sleep disorder and others. FKS constitutes a robust and objective measure of changes in motor performance. Therefore, the FKS allows correct identification of motor fatigue even in cases where common comorbidities mask motor fatigue.

Keywords Fatigue - Gait analysis - Fatigue index · Physical performance $\cdot$ Lactate level $\cdot$ Heart rate

\section{Introduction}

Fatigue is a common and frequently disabling symptom of multiple sclerosis (MS) with profound negative effects on normal activities of daily life and substantially reduces quality of life [1]. Fatigue is associated with a subjective feeling of weakness, lack of energy and lethargy [2]. Between 38 and $83 \%$ of patients with MS suffer from fatigue [3], and many patients report fatigue to be their most debilitating symptom [2]. However, despite the high incidence of fatigue, its pathophysiology is still poorly understood. Many factors have been proposed to play a role in the development of fatigue in MS [4]. Two distinct types of fatigue in MS - mental and motor fatigue - can occur in isolation or in combination. Psychological and/or physical condition might be compromised by factors such as depression, sleep disorder and deconditioning, which may influence the symptoms of fatigue [2]. Further, fatigue may be caused by a failure of the neuromuscular system to 
generate force [5]. Patients with MS show significantly prolonged central motor conduction times and lower motor evoked potential amplitudes following fatiguing exercise [6]. Fatigue may be caused by an intermittent central usedependent conduction block [7], and metabolic abnormalities in the frontal cortex and basal ganglia have been observed via positron emission tomography in MS patients with fatigue [8].

In the current study, we focused on the motor dimension of fatigue. Presently, there is no "gold standard" for detecting motor fatigue. Commonly, fatigue is assessed by several questionnaires including the Fatigue Severity Scale (FSS) [9], the Fatigue Assessment Instrument (FAI) [10], the Fatigue Impact Scale (FIS) [11], the Modified Fatigue Impact Scale (MFIS) [12] and, in Germany, the Fatigue Scale for Motor and Cognitive Functions (FSMC) [13] and the Würzburg Fatigue Inventory in Multiple Sclerosis (WEIMuS) [14]. These standardized questionnaires are based on patients' self-assessments and capture their general condition during a particular time course. However, because these questionnaires are based on the patients' subjective feelings, they may be distorted (overestimation or underestimation) due to an inaccurate self-perception. In some patients with MS, deciding whether motor fatigue is present or whether other factors cause similar symptoms can be very difficult. Hence, an objective tool for assessing motor fatigue in MS is crucial for a more precise diagnosis of fatigue and for the design and evaluation of treatment and rehabilitation programs.

Recently, we have introduced an unbiased instrument for assessing motor fatigue [15]. Specific gait parameters including step length, width and height, bilateral leg circumduction, bilateral knee flexion angle and medio-lateral trunk sway changed during a walking test. The step cycle was more irregular at the end compared with the beginning of the test. These changes in gait patterns were found to be symptomatic for MS patients with motor fatigue. Such traditional kinematic analyses of human gait capture the characteristic pattern for an individual based on a few single gait cycles $[16,17]$ but neglect essential information of the dynamics of continuous locomotion [17]. Non-linear methods use gait data in a time series format to calculate and interpret the Lyapunov exponent as an estimator for dynamic stability $[18,19]$. Although this method may have its advantages, the results can be well presented only at the group level and not at the individual patient level [20]. This must be considered in clinical trials and for decisionmaking in clinical practice.

We have recently presented a new method for quantifying differences in time series of gait data using attractor attributes with an underlying limit cycle attractor [21]. Using this method, we were able to identify subtle gait pattern differences between different situations and classify these on group and subject levels. A stable situation is characterized by an unchanged attractor and unchanged movement variability. Thus, changes of these two parameters indicate an alteration of the gait mechanism, which, by ruling out other reasons, we identify as acute motor fatigue.

To the best of our knowledge, an objective method for classifying fatigue in patients with MS is lacking. Therefore, the purpose of this study was to establish the "Fatigue index Kliniken Schmieder (FKS)" as an objective numerical indicator for assessing motor fatigue using kinematic gait analysis and to test its sensitivity and specificity. We hypothesized that the FKS allows precise identification of subjects with motor fatigue and that motor fatigue in MS is not related to muscle fatigue.

\section{Materials and methods}

\section{Subjects}

Twenty healthy subjects and forty patients with definite MS according to the McDonald criteria [7] participated in this study (Table 1). Patients and controls were all between 18 and 65 years old and recruited between October 2011 and July 2012. All patients were admitted to the inpatient rehabilitation clinic specializing in MS; and had to be able to walk on a treadmill without aids or assistance. There were no limitations of disease course and disability levels. Patients were in a stable condition and those with relapses

Table 1 Mean (1 standard deviation) subject characteristics

\begin{tabular}{llll}
\hline Parameters & $\begin{array}{l}\text { Patients with } \\
\text { MS }\end{array}$ & $\begin{array}{l}\text { Healthy } \\
\text { subjects }\end{array}$ & $P$ value \\
\hline Sex (female/male) & $27 / 13$ & $11 / 9$ & n.s. \\
Age (years) & $45.9(7.0)$ & $43.1(8.6)$ & n.s. \\
Height $(\mathrm{cm})$ & $171.3(10.7)$ & $173.4(8.4)$ & n.s. \\
Mass (kg) & $74.1(15.6)$ & $80.4(21.3)$ & n.s. \\
Disease course $(n)$ & & Not & \\
RR & 30 & applicable & \\
& & Not & \\
PP & 3 & applicable & \\
& & Not & \\
SP & 7 & applicable & \\
EDSS & $3.4(1.3)$ & Not & \\
& & applicable & \\
BDI-II $>8$ ( $n$ with & 26 & 3 & 0.001 \\
depression) & & & \\
\hline
\end{tabular}

$M S$ multiple sclerosis, $R R$ relapsing-remitting, $P P$ primary progressive, $S P$ secondary progressive, EDSS Expanded Disability Status Scale, BDI-II Beck Depression Inventory, $n$ number of subjects, $P$ value probability value, n.s. not statistically significant 
within the past 3 months were excluded. Subjects were also excluded from the study if they received the drug Fampyra $^{\circledR}$ (Fampridin; Biogen Idec Inc., 225 Binney Street, Cambridge, MA 02142, USA). Participants were consecutively enrolled upon their willingness to partake in this study. Control subjects were recruited from the local population and from clinic staff. The healthy subjects were included in the study by volunteering and if they had no neurological or orthopedic disorders.

\section{Exercise task}

All participants completed a walking test on a treadmill: they walked either until they experienced complete exhaustion (17, very hard on the Borg scale [22]) or for a maximum of 60 min. Based on our previous results [15], we expected that the MS patients with fatigue will be exhausted within substantially $<60 \mathrm{~min}$. All subjects wore a harness during the walking test to prevent falling. The treadmill speed was set to $10 \%$ above a subject-specific comfortable walking speed or to a maximum of $5 \mathrm{~km} / \mathrm{h}$ and kept constant throughout the test. During the test, participants were repeatedly asked to rate their exhaustion on a Borg scale from 6 (absolutely no effort) to 20 (maximum effort). The test was stopped one minute after the patient reached 17 on the Borg scale ("very hard") or after 60-min walking.

Gait data were recorded for $1 \mathrm{~min}$ at the beginning $\left(t_{1}\right)$ and for $1 \mathrm{~min}$ at the end of the test $\left(t_{2}\right)$ using the motion analysis device AS200 (LUKOtronic, Lutz Mechatronic Technology e.U., Innsbruck, Austria; sampling rate $80 \mathrm{~Hz}$ ). Markers were placed bilaterally on the heel counter of the shoes; bilaterally with a rod on the distal portion of the Achilles tendon at the level of the ankle; bilaterally on the posterior aspect of the knee; bilaterally on the belt at the highest point of the iliac crest; and bilaterally centered on the margo medialis. Accelerations of the markers were computed as the second derivatives of the position traces after filtering with an $F^{3}$-filter at $4.5 \mathrm{~Hz}$ cutoff [23].

Blood samples were taken prior to $t_{1}$ and immediately after $t_{2}$. Blood lactate levels were measured using a lactate analyzer and lactate strips with a precision of $3 \%$ coefficient of variation (Arkray Lactate Pro LT-17810, Kyoto, Japan). Heart rate was measured prior to $t_{1}$ and at $t_{2}$ using a chest strap and a heart rate monitor (Garmin Forerunner 305, Garmin Ltd., Kansas City, Kansas, USA).

Diagnostic criteria

\section{Theoretical background}

Human walking in the absence of disturbances is characterized by a stable movement pattern and consistent movement control. Because we kept the walking situation unchanged and muscular exhaustion was excluded (lactate assessment), pattern and variability changes during the walking test were deemed to have been caused by fatigue. We applied a new method [21] for quantifying movement pattern changes and changes of movement variability using attractor attributes of the acceleration of the feet. The limit cycle attractors were approximated and three measures were calculated: $\delta M$ is a measure of the difference between two attractors quantifying the differences between two movement patterns; $\delta D$ is the difference between the two associated deviations of the state vector away from the attractor representing the change in movement variation; and $\delta F$ is the product of $\delta M$ and $\delta D$ and represents an index of the change which we termed the FKS.

$\delta F=\delta M \cdot \delta D$

Three-dimensional acceleration measurements $\left(\vec{a}_{r}(t)\right.$, $\left.\vec{a}_{l}(t)\right)$ of two markers (1, left foot; $\mathrm{r}$, right foot) were used. The start and the end of a loop were defined as the passing of $\vec{a}_{r}(t)$ respectively $\vec{a}_{l}(t)$ through a well-defined 2D area (Figs. 1a, b, 2a, b). The attractor parameters $\left(\vec{A}_{r}(\tau), \vec{A}_{l}(\tau)\right)$ were approximated as the acceleration at time $\tau$ (ordering parameter of a complete cycle) averaged over $n$ loops.

$$
\begin{aligned}
\vec{A}_{\mathrm{a}, \mathrm{C}}\left(\tau_{j}\right) & =\frac{1}{n} \sum_{i=1}^{n} \vec{a}_{\mathrm{a}, \mathrm{C}}\left(i \cdot \tau_{j}\right)+\frac{1}{n} \sum_{i=1}^{n} \vec{b}_{\mathrm{a}, \mathrm{C}}\left(t=i \cdot \tau_{j}\right) \\
& \approx \frac{1}{n} \sum_{i=1}^{n} \vec{a}_{\mathrm{a}, \mathrm{C}}\left(i \cdot \tau_{j}\right)
\end{aligned}
$$

with

$$
\lim _{n \rightarrow \infty} \frac{1}{n} \sum_{i=1}^{n} \vec{b}\left(i \cdot \tau_{j}\right)=0
$$

and $a=$ right or left and $C=$ beginning or end.

$\left(\vec{D}_{r}(\tau), \vec{D}_{l}(\tau)\right)$ are the standard deviations describing differences of the actual acceleration and the attractor.

$D_{\mathrm{a}, \mathrm{C}}\left(\tau_{j}\right)=\sqrt{\frac{1}{n-1} \sum_{i=1}^{n}\left[\vec{A}_{\mathrm{a}, \mathrm{C}}\left(\tau_{j}\right)-\vec{a}_{\mathrm{a}, \mathrm{C}}\left(i \cdot \tau_{j}\right)\right]^{2}}$

Differences in gait parameters between the beginning $(B)$ and the end $(E)$ of the walking test were calculated as $\delta M=\frac{1}{v} \sqrt{\sum_{i=1}^{3}\left[\left\langle\left(A_{r, B, x_{i}}-A_{r, E, x_{i}}\right)^{2}\right\rangle+\left\langle\left(A_{l, B, x_{i}}-A_{l, E, x_{i}}\right)^{2}\right\rangle\right]}$

The change in the standard deviation of attractor and acceleration measurements was defined as 

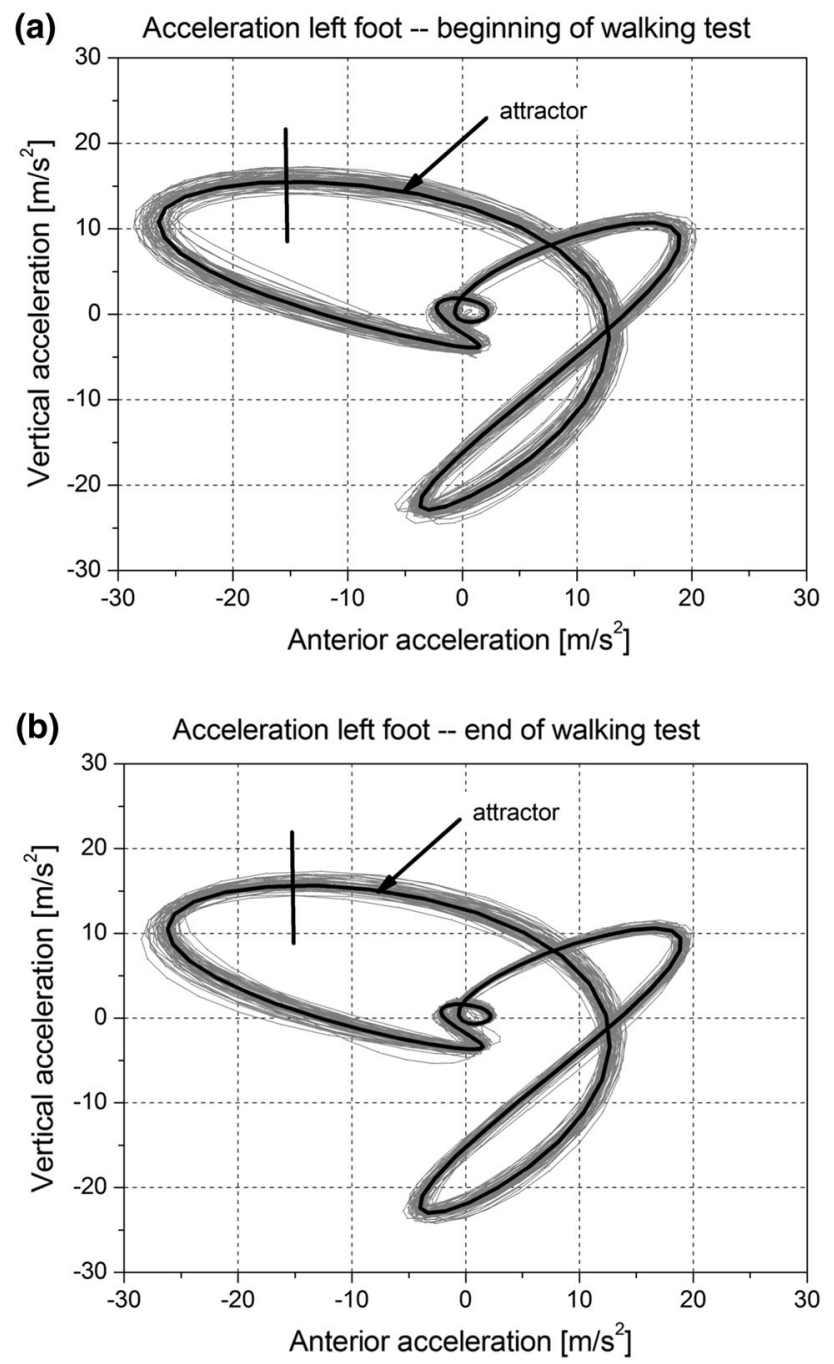

Fig. 1 Two-dimensional graph of the acceleration data of a subject's left foot for $1 \mathrm{~min} \mathbf{a}$ at the beginning and $\mathbf{b}$ at the end of the walking task for one healthy subject. The vertical black line indicates the plane that was used to define the beginning and end of each gait cycle

$\delta D=\sqrt{\left\langle\left(D_{r, B}-D_{r, E}\right)^{2}\right\rangle+\left\langle\left(D_{l, B}-D_{l, E}\right)^{2}\right\rangle}$

where $\langle\ldots\rangle$ is the average of the included expression.

\section{Deriving the Fatigue index Kliniken Schmieder (FKS)}

The FKS is based on the two attractors calculated from the three-dimensional acceleration data of the feet of a walking person. Non-changing attractors define a stable system. The FKS allows for capturing altered movement patterns and variability on an individual level representing fatigability. To calibrate the FKS, we used the method described in [21] requiring data of two groups of subjects as selected by a conventional method. Central to this method is the median (a) Acceleration left foot -- beginning of walking test

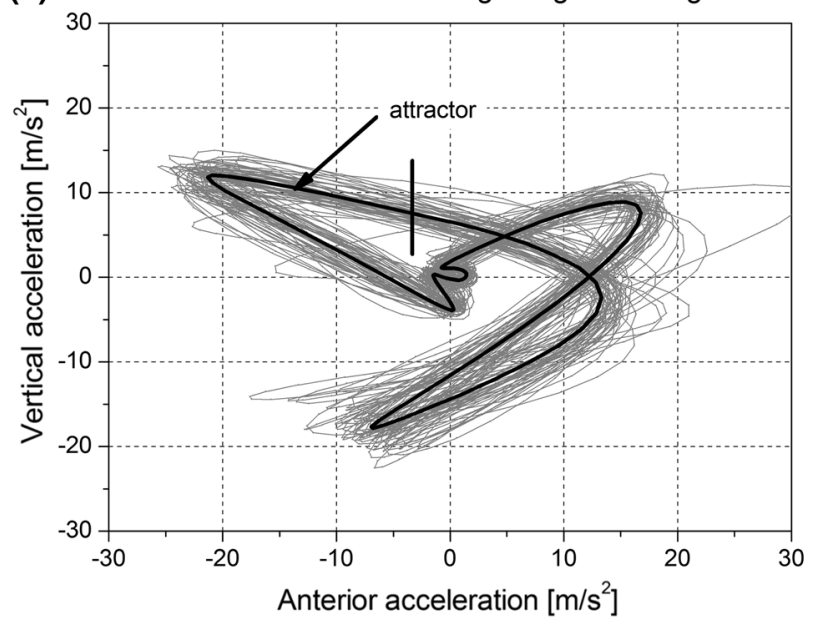

(b) Acceleration left foot -- end of walking test

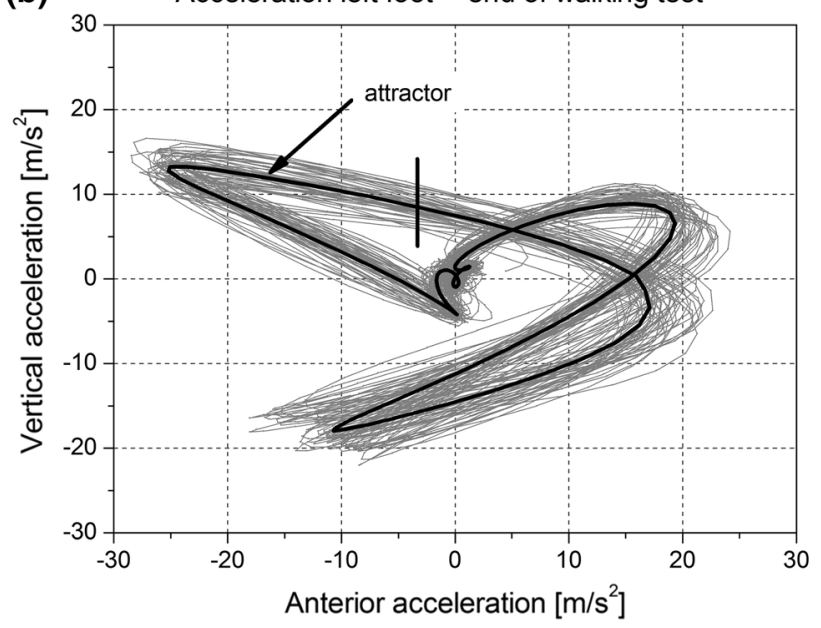

Fig. 2 Two-dimensional graph of the acceleration data of a subject's left foot for $1 \mathrm{~min} \mathbf{a}$ at the beginning and $\mathbf{b}$ at the end of the walking task for one MS patient with fatigue. The vertical black line indicates the plane that was used to define the beginning and end of each gait cycle

of the two groups, which are not significantly influenced by outliers. Conventional methods allow clear identification of the most typical group members; e.g. when looking for fatiguing MS patients it is easy to identify heavily fatigued subjects. Those individuals with mild symptoms might be wrongly classified as not fatiguing. Similarly non-fatiguing persons with walking constraints have a high probability of wrongly being included into the fatigue group. As long as a group does not contain more than $25 \%$ wrongly sorted subjects and both groups have about the same ratio of wrong members, it would not substantially influence the FKS-medians of the groups. The next step in the calibration process is to calculate the lower $\alpha$-quantile of the fatigue group and the upper $\alpha$-quantile of the non-fatigue group. We increased the $\alpha$ until the maximal non-overlapping 


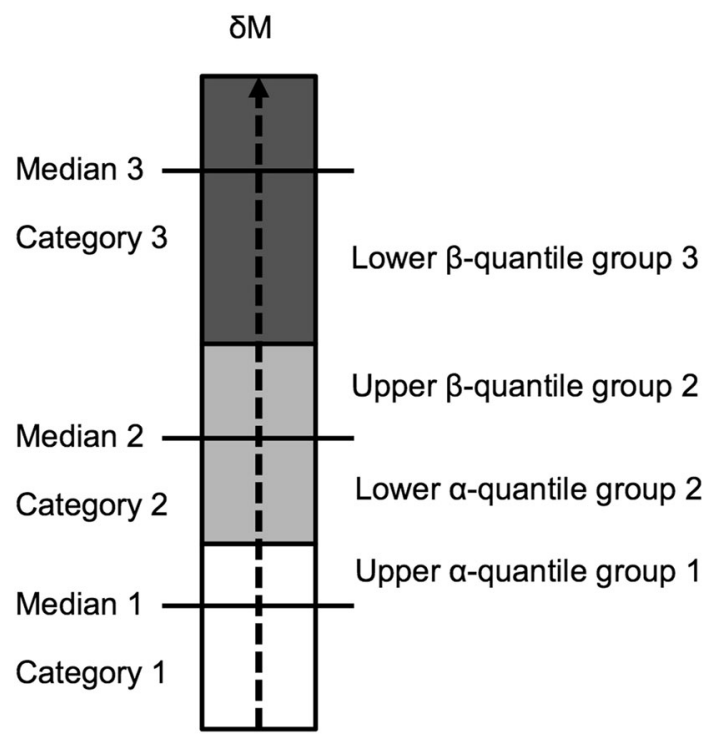

Fig. 3 "Illustration of example categories used to allow the creation of Quantiles from the medians of different groups" [21]

quantiles were identified (Fig. 3) [21]. The nearest integer within the interval defined by the smallest FKS-value in the lower $\alpha$-quantile (fatigue group) and the largest FKS-value in the upper $\alpha$-quantile (non-fatigue group) established the limit between fatigue and non-fatigue.

Next, the FKS of healthy individuals was used as a benchmark test; and following, all subjects were classified according to the FKS values into the fatigue and the nonfatigue groups. All further analyses were performed for three groups: patients with fatigue symptom (MS-F), patients without fatigue symptom (MS-NF) and healthy persons (non-MS). The results of a "conventional" instrument (physician's judgment, physiotherapists' rating, fatigue questionnaire) were compared to those of the new instrument (FKS). Also, the effect of disease course or Expanded Disability Status Scale (EDSS) scores [24] on FKS was investigated. Finally, we detected the differences between groups' movement patterns as well as in their physical performance.

\section{Assessment by the neurologist}

The objective measure of motor fatigue was calibrated using a neurologist's judgment of fatigue presence. As explained above the calibration is based on the most obvious fatiguing subjects being classified into the fatigue group and the clearly non-fatiguing members in the nonfatigue group. The judgment of the "critical cases", those which are not clearly classifiable, cannot uniquely be rated by experts; FKS, however, gives definite results.

The neurologist sorted MS patients into two groups: patients with fatigue symptom and patients without fatigue symptom. Motor fatigue was assumed if the patient reported an abnormal rapid physical exhaustion in daily living and if a severe reduction in maximum gait distance could not be explained by the degree of paresis, spasticity or ataxia. The neurologist (C.D.) was not aware of the results of the walking test, which was used to establish the numerical base of the FKS calculation. The researcher (A.S.) performing the walking test did not have any knowledge of the neurologist's assessment.

\section{Video recordings}

Videos of the subjects while walking were recorded and shown to experienced physiotherapists. The physiotherapists were given simultaneously recorded side and back views of the subjects. Two raters (employees of the rehabilitation clinic) who were unaware of the FKS analysis and the neurologist's assessment rated the video recordings of the subjects while walking on the treadmill. For each participant, videos of the beginning and the end of the test were shown to the raters in random sequence. Raters were asked to assign the videos of each subject to the beginning or the end of the walking test.

\section{Questionnaires}

All patients completed questionnaires for assessing dimensions of subjective cognitive, motor and total fatigue (FSMC, the Fatigue Scale for Motor and Cognitive Functions [13]) and for measuring the degree of subclinical depression according to the beck depression inventory II (BDI-II) $[25,26]$. The healthy volunteers only completed the BDI-II.

\section{Conventional gait analysis}

Spatial parameters were calculated for each gait parameter and time interval for each subject using the three-dimensional coordinates of the infrared markers. The following gait parameters were assessed and compared between groups: step length, step width, step height, maximum circumduction with the right and left leg, maximum knee flexion angle of the right and left leg, and medio-lateral sway of the upper body.

Functional tests

Walking distance, walking velocity, lactate level, and heart rate as well as the degree of exertion on the Borg scale during the walking test were recorded. All subjects completed a 6-min walk test (6MWT) [27]. The 6MWT is safe, easy to administer, well-tolerated, and reflects the ability to perform activities of daily living [28]. A reduced distance 
during the 6MWT indicates impairment and is often used in clinical practice [28].

Statistical methods

All statistical tests were performed using StatFree Version 7.0.2.3 (VietenDynamics, University of Konstanz, Germany) and Stata Version 11.0 (StatCorp LP, College Station, Texas, USA). The null hypothesis for each analysis was rejected at $p<0.05$. Differences in parameters between groups and between time points were detected using analysis of variance (ANOVA). Bonferroni post hoc tests were used to detect significant differences between groups. Differences in FKS values between the three groups were identified using Student's $t$ tests for independent samples. Variations in non-normally distributed parameters between groups were detected using KruskalWallis test with Mann-Whitney $U$ test as post hoc tests. For binary variables, we used the Cohen's Kappa test to measure agreement between raters. For categorical variables we used the $\chi^{2}$-test. Pearson correlation coefficients were used to detect significant associations between the components of the FKS, the dimensions of fatigue questionnaires and the EDSS. Multiple regression analysis was used to detect the effect of disease course and EDSS scores on the FKS.

\section{Results}

\section{Calibrating of the FKS}

With the conventional method (neurologist rating) eleven patients with MS were categorized as not having fatigue. They together with the healthy subjects displayed an FKSmedian of 0.9. The fatigue group, as identified conventionally, consisted of 29 MS patients having an FKSmedian of 9.5. The largest non-overlapping quantiles were $46 \%$, where the largest FKS value of the upper quantile (non-fatigue group) was 3.9 and the smallest value of the lower quantile (fatigue group) was 4.2. The nearest integer " 4 " was established as the boundary between non-fatigue and fatigue (Table 2). The FKS between 0 and 4 points represented the "normal range". Quantiles of $50 \%$ would have meant identical selections by the traditional method and by using FKS. Quantiles of $46 \%$ indicate wrong allocations, two cases as we found out. However, as detailed in the methodology, the boundary " 4 " between the non-fatigue and the fatigue group is not substantially influenced by up to $25 \%$ wrongly sorted subjects (mildly fatiguing subjects within the non-fatigue group and persons with walking constraints within the fatigue group). To test
Table 2 Classification of subjects into groups

\begin{tabular}{lllll}
\hline FKS & Classification & MS-F $(n)$ & MS-NF $(n)$ & Non-MS $(n)$ \\
\hline$\leq 4$ & No fatigue & & 11 & 20 \\
$>4$ & Fatigue & 29 & & \\
\hline
\end{tabular}

$F K S$ the fatigue index Kliniken Schmieder, $M S-F$ patients with multiple sclerosis and fatigue symptom, $M S-N F$ patients with multiple sclerosis and without fatigue symptom, Non-MS healthy persons, $n$ number of subjects

this we deliberately shifted the four subjects with the highest FKS from the non-fatigue group into fatigue group and four with the lowest FKS from the fatigue into the nonfatigue group. By using the same calculation procedure we obtained medians 0.9 and 8.8 respectively. Here the maximal non-overlapping quantiles were found at $35 \%$ with 3.9 as the largest FKS value in the non-fatigue selection and the smallest value of the lower quantile (fatigue group) as 4.2. This is the same result as before with exactly the same "nearest integer" as the boundary value between non-fatigue and fatigue in spite of the bigger number of wrongly sequenced subjects.

\section{Benchmark test}

All healthy subjects' FKS were below the threshold of "4", which discriminates between subjects without and with fatigue. The median FKS in healthy subjects was 0.7 (range $0.3-3.9$ ), $\delta M$ ranged from 0.6 to 4.3 and $\delta D$ from 0.3 to 1.5 . All healthy subjects walked for $60 \mathrm{~min}$ and had maximum scores below 17 on the Borg scale.

Rearranging the MS patients using the FKS results

The MS-F group had a FKS range from 4.2 to $125, \delta M$ between 2.8 and 30.4, and $\delta D$ between 0.9 and 4.1. The MS-NF group had a range of FKS from 0.5 to $3.4, \delta M$ between 1 and 3.6, and $\delta D$ between 0.4 and 1.0. The FKS differed significantly between the MS-F and the MS-NF and non-MS groups $(p<0.001)$ as expected based on the definition of fatigue, but not between the MS-NF group and the non-MS group $(p=0.69)$. Patients with fatigue showed greater changes in movement pattern and variability during the test than patients without fatigue and healthy subjects (Fig. 4).

Age, gender distribution, body height and weight did not differ between the MS-F and the MS-NF groups ( $p \geq 0.51$ ) (Table 3). Moreover, patients in both groups were similarly affected by depression and there was no significant difference in the course of the disease $(p \geq 0.51)$. However, the MS-F group had significantly longer disease duration and greater EDSS and FSMC scores than the MS-NF group $(p \leq 0.03)$. 


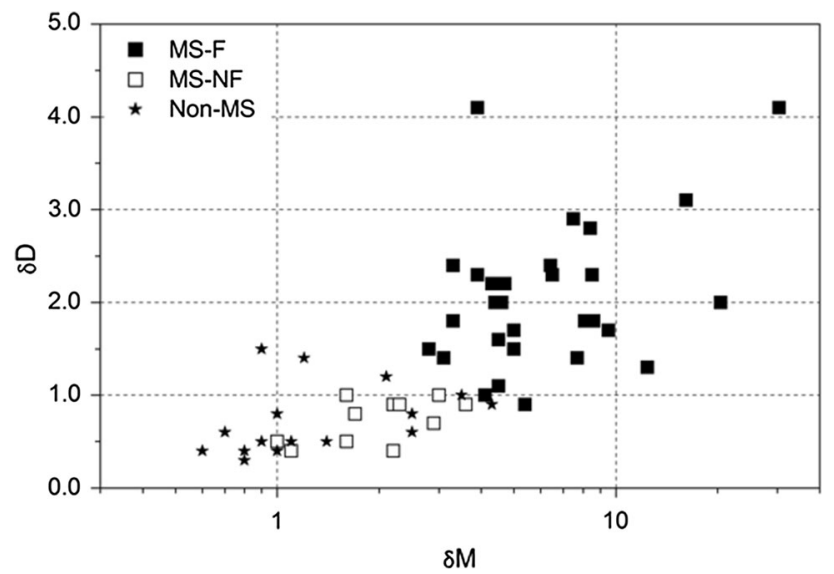

Fig. $4 \delta M$ vs $\delta D$ for all subjects. MS patients with fatigue, MS patients without fatigue and healthy subjects are clearly separated. A logarithmic scale was used for the $\delta M$-axis to account for the large differences in $\delta M$ between and within groups

Table 3 Mean (1 standard deviation) characteristics of MS patients with fatigue (MS-F) and MS patients without fatigue (MS-NF)

\begin{tabular}{llll}
\hline Parameters & MS-F & MS-NF & $P$ value \\
\hline Sex (female/male) & $19 / 10$ & $8 / 3$ & n.s. \\
Age (years) & $45.3(7.0)$ & $46.6(7.6)$ & n.s. \\
Height (cm) & $170.6(10.3)$ & $173.3(11.9)$ & n.s. \\
Mass (kg) & $72.5(13.9)$ & $78.0(19.6)$ & n.s. \\
Disease duration (years) & $12.3(7.3)$ & $6.7(5.4)$ & 0.03 \\
EDSS & $3.8(1.2)$ & $2.4(1.1)$ & 0.004 \\
Disease course $(n)$ & & & n.s. \\
$\quad$ RR & 20 & 10 & \\
PP & 2 & 1 & \\
$\quad$ SP & 7 & 0 & \\
FSMC motor dimension score & $38.6(8.1)$ & $27.4(10.0)$ & 0.002 \\
BDI-II (\% of depression) & 65.5 & 63.4 & n.s. \\
\hline
\end{tabular}

$M S$ multiple sclerosis, EDSS Expanded Disability Status Scale, $n$ number of subjects, $R R$ relapsing-remitting, $P P$ primary progressive, $S P$ secondary progressive, FSMC fatigue scale for motor and cognitive functions, BDI-II Beck Depression Inventory, P-value probability value, n.s. not statistically significant

Comparison of FKS results, assessment by the physician, and video analysis through physiotherapists

Classification of fatigue using the FKS and the neurologist's assessment was consistent for $97 \%$ (false positive of $3 \%$ ) for the fatigue group; and $91 \%$ (false negative of $9 \%)$ for non-fatigue. One patient was classified into the fatigue group by the neurologist but did have a FKS of 2.1, a clear indication of no fatigue. Another patient had a FKS of 11-massively fatiguing - but was classified into the no fatigue group by the neurologist.
Both physiotherapists correctly classified 56 of 58 (97\%) videos in the MS-F group. After the test, they reported that they could not see any difference in the two misclassified videos. For the non-fatiguing and healthy subjects the videoclassification would simply show random results-having a $50 \%$ chance for correct classification. One physiotherapist classified 12 of 22 videos and the other physiotherapist 8 of 22 videos correctly for the MS-NF group. In the group of healthy subjects the physiotherapists correctly classified 26 of 40 and 34 of 40 videos, respectively.

We compared the results of the subjective assessment by physician and physiotherapists to the results of the objective measure FKS and found overall good agreement but faulty classifications as well. The accordance between the physician's assessment and FKS were good (Cohen's Kappa: $\kappa=0.88$ ), between physiotherapists' assessment and FKS (Cohen's Kappa: $\kappa$ from 0.32 to 0.49 ) fair to moderate agreement.

FKS, EDSS and questionnaires

In the MS-F group, the FKS and $\delta M$ did not correlate significantly with any dimensions of the FSMC or EDSS (range $r-0.05$ to $0.20 ; p>0.13$ ). $\delta D$ correlated negatively with all dimensions of the FSMC (range $\mathrm{r}-0.39$ to -0.28 ; $p<0.03)$ and positively with the EDSS $(r=0.28$; $p<0.03$ ). In the MS-NF group, the FKS and $\delta M$ correlated negatively with all dimensions of the FSMC (range $r-0.67$ to $-0.57, p<0.006)$. $\delta D$ correlated with the overall and motor dimensions of the FSMC $(r=0.42 ; p<0.04)$ but not with the cognitive score of the FSMC $(r=-0.39$; $p>0.07)$. None of the components of the FKS correlated with the EDSS (range $r-0.35$ to $0.31 ; p>0.11$ ).

Multiple linear regression analysis did not reveal significant relationships between disease course $(p=0.40)$ and EDSS scores $(p=0.11)$ and FKS (model: adjusted $\left.R^{2}=0.03, p=0.25\right)$. Correlations between EDSS and FKS for each disease group revealed an interaction effect: while there is a small correlation between the two measures in the group with relapsing-remitting disease course $(r=0.33 ; p \leq 0.01)$, the correlation in the group with secondary progressive disease course is nearly zero $(r=0.05 ; p=0.86)$.

Gait analysis

The MS-F group proved to be significantly different from the MS-NF and healthy groups in all gait parameters including step length, width and height, bilateral leg circumduction, bilateral knee flexion angle and medio-lateral trunk sway at $t_{1}$ and $t_{2}(p<0.001)$. Patients in the MS-F group had smaller step length, step height and maximum knee flexion angle on the right and left side and greater step 
Table 4 Mean (1 standard deviation) gait and physiological parameters of the treadmill walking test for MS patients with fatigue (MS-F), MS patients without fatigue (MS-NF) and healthy subjects (non-MS)

\begin{tabular}{|c|c|c|c|c|c|}
\hline Parameters & MS-F & MS-NF & Non-MS & $\begin{array}{l}P \text { value Kruskal- } \\
\text { Wallis test }\end{array}$ & $\begin{array}{l}P \text { value Post } \\
\text { hoc test }\end{array}$ \\
\hline Walking distance $(\mathrm{km})$ & $1.8(1.2)$ & $4.4(1.2)$ & $5.3(0.3)$ & 0.001 & $\begin{array}{l}0.001^{*} \\
0.001^{\dagger} \\
0.012^{*}\end{array}$ \\
\hline Walking speed $(\mathrm{km} / \mathrm{h})$ & $0.81(0.36)$ & $4.7(0.5)$ & $5.0(0.0)$ & 0.001 & $\begin{array}{l}0.001^{*} \\
0.001^{\dagger} \\
0.001^{*}\end{array}$ \\
\hline 6MWT (km) & $0.49(0.09)$ & $0.56(0.12)$ & $0.68(0.10)$ & 0.001 & $\begin{array}{l}0.008^{*} \\
0.001^{\dagger} \\
0.011^{*}\end{array}$ \\
\hline \multicolumn{6}{|l|}{ Lactate $(\mathrm{mmol} / \mathrm{l})$} \\
\hline $\begin{array}{l}t_{1} \\
t_{2}\end{array}$ & $\begin{array}{l}1.2(0.5) \\
0.7(0.5)\end{array}$ & $\begin{array}{l}0.9(0.6) \\
0.4(0.5)\end{array}$ & $\begin{array}{l}0.8(0.6) \\
0.6(0.6)\end{array}$ & $\begin{array}{l}\text { n.s. } \\
\text { n.s. }\end{array}$ & \\
\hline \multicolumn{6}{|l|}{ Heart rate (bpm) } \\
\hline $\begin{array}{l}t_{1} \\
t_{2}\end{array}$ & $\begin{array}{r}80.0(11.3) \\
103.7(18.2)\end{array}$ & $\begin{array}{r}77.0(10.4) \\
107.5(12.8)\end{array}$ & $\begin{array}{r}79.4(20.7) \\
108.8(20.8)\end{array}$ & $\begin{array}{l}\text { n.s. } \\
\text { n.s. }\end{array}$ & \\
\hline Borg scale & $16.9(0.4)$ & $13.5(4.0)$ & $10.0(2.5)$ & 0.001 & $\begin{array}{l}0.001^{*} \\
0.001^{\dagger} \\
0.007^{\star}\end{array}$ \\
\hline
\end{tabular}

6MWT 6-min walk test, n.s. not statistically significant

* Significantly different between MS-F and MS-NF

$\dagger$ Significantly different between MS-F and non-MS

* Significantly different between MS-NF and non-MS, $P$ value probability value fatigue using the FKS and the classification by the neurologist and two physiotherapists. We found FKS to be a more sensitive and reliable measure for motor fatigue in patients with MS compared to traditional methods. FKS is firmly based on the definition of "system stability". For calibration it uses the "medians" of classically defined subject groups, a parameter largely insensitive to incorrect subject selection. Lactate levels below the lactate threshold in all patients and subjects suggest that motor fatigue is not related to muscle fatigue.

The classification into fatigue and non-fatigue groups using the FKS versus the classification by the neurologist disagreed for two patients. In one patient, the diagnosis of MS was very recent (primary progressive course of disease). She complained of parasthesia in her right leg, reported that she got tired more easily than previously and described her walking distance as reduced. In the neurological exam, she had difficulty jumping on her right leg and a tendency to pronate her left arm during arm holding. In addition, her motor FSMC was 27 (moderate motor fatigue). The treadmill test clearly showed that she had no motor fatigue $(4.4 \mathrm{~km}$ in $60 \mathrm{~min})$. Hence, this patient was likely misclassified by the traditional classification system suggesting FKS was superior to traditional assessment.

Another patient classified as not having fatigue by the neurologist showed a high FKS. This patient suffered from severe depression with apathy and sleep disorder making the additional clinical diagnosis of motor fatigue difficult. The pathological FKS, however, clearly demonstrated were moderate differences between the classification of 
increased variability while walking indicating motor fatigue.

A recent study [29] has shown that reduced sleep efficiency on nocturnal polysomnographic findings correlate with fatigue, tiredness, and lack of energy in patients with MS. Labuz-Roszak et al. [30] observed an association between fatigue and sleep disorders and between fatigue and either depression or anxiety, and stated that the treatable causes of fatigue in MS such as sleep and mood disturbances should be identified and treated. The results of our study clearly indicate the presence of additional motor fatigue besides the presence of sleep disorder and depression, which cannot be identified with certainty on clinical grounds without an objective measure of fatigue. These observations further confirm the importance of an objective measure for assessing motor fatigue in patients with MS.

None of the patients in our study reported absence of motor fatigue and were tested positive on the FKS. We would consider such cases rather rare. Nonetheless, we believe that a patient without subjective complaints of motor fatigue should not be treated for motor fatigue.

The results of the FKS largely agreed with the results of the video analysis. Both physiotherapists assigned videos of the beginning and end correctly for $97 \%$ of patients with fatigue. Such classification was almost impossible for healthy subjects or patients without fatigue. Even if this evaluation was very successful for these cases, this analysis is subjective and depends on many factors and in particular on the therapists' experience. These results further emphasize the value and the effectiveness of an objective measure of motor fatigue using FKS.

We consider the FKS superior compared to traditional methods because of its unambiguous approach and objectivity. Overall, the diagnosis of fatigue conducted by physicians or physiotherapists may be faster, but also more likely to give false results. Practical experience demonstrates that a precise diagnosis of fatigue and non-fatigue depends on the experience of the therapist and physician. It is possible that experienced neurologists and physiotherapists may be correct in their classification in most MS patients. However, some neurologists with no or little experience with MS patients may doubt the existence of fatigue and its organic cause; and thus may argue that fatigue just reflects the difficulty of the patient to cope with MS. In some cases, identifying motor fatigue may be very difficult even for an expert; especially if depression, sleep disturbances, adiposity and lack of exercise and endurance are also prevalent. In these cases, identifying different components of the patients' symptoms is very helpful for the therapist, neurologist and patient and is important for the "therapeutic alliance" or "therapeutic contract" between patient and therapist.

Our observations of differences in conventional gait patterns in the MS-F group compared to the MS-NF and healthy group are in agreement with literature. Previous studies [3135] have reported reduced gait speed, reduced stride length, step width and maximum knee extension as well as greater trunk angle range of motion in patients with MS compared with healthy subjects. However, the authors of these studies did not distinguish between patients with fatigue and those without fatigue, and there is no previous data on changes in gait patterns dependent on fatigue. In comparison, our novel classification method can attribute changes in gait patterns to the presence or absence of fatigue.

The overall and motor dimensions of self-rated fatigue questionnaires did not correlate with the FKS in the MS-F group. These findings are in agreement with earlier reports of no relation between changes in walking patterns and selfassessed fatigue [34]. Morris et al. [34] suggested that mechanisms controlling locomotion differ from those regulating perceived fatigue. Perceived fatigue may not be associated with performance in conventional neuropsychological tests or motor function tasks [36-38]. Kluger et al. [3] emphasized the importance of distinguishing between subjective sensation and objective changes of performance representing fatigability. In this context, the scientists used the term "fatigability" rather than "fatigue syndrome". This is also in line with the current concept that fatigue has a state and trait component [39]. Genova et al. [39] defined "state fatigue" as a transient condition that can fluctuate based on both external and internal factors and "trait fatigue" as a more stable condition in an individual. According to this definition, the FSMC and other questionnaires might capture the trait component of fatigue; while the FKS represents the state component of fatigue after physical exertion. Our parameters allow statements regarding the changes in movement pattern and changes in movement variability after exhausting or prolonged walking. Hence, these values measure a patient's current state of fatigue in contrast to the FSMC questionnaire, which presumably captures a patient's general physical and mental condition. This indicates that the fatigue questionnaire and the FKS record two different features of fatigue.

We found no evidence of an effect of disease course or EDSS scores on FKS. The overall model fit was very low, indicating that FKS measures fatigue differently than other existing measures.

All participants in our study remained below the anaerobic threshold throughout the walking test and had only slight changes in heart rate between $t_{1}$ and $t_{2}$ suggesting that motor fatigue in MS is not related to muscle fatigue. In contrast, Sharma et al. [40] reported that during exercise phosphocreatine and intracellular $\mathrm{pH}$ was greater in patients with MS than in controls. Simultaneously, during the exercise inorganic phosphate and monovalent inorganic phosphate increased more in patients with MS than in healthy persons. They suggested that metabolism 
plays a role in abnormal muscle fatigue in MS. The discrepancy between our results and those reported by Sharma et al. may be related to differences in EDSS scores in both cohorts [Sharma et al. 5.1 (range 2-8), 20 of 28 patients walked with a cane, walker or were wheelchair-bound; our study 3.4 (range 1-6.5), no walking aids] and to the use of intermittent tetanic stimulation of the peroneal nerve versus walking exercise. The walking test in our study may better resemble daily activities while being standardized and reproducible.

In summary, we have developed an objective method for assessing motor fatigue on an individual basis using kinematic gait analysis-the FKS. It correctly classified patients with MS into fatigue and non-fatigue groups. This method represents a quick, cost-effective, and efficient method for objectively assessing motor fatigue. The FKS did not correlate with the results of the fatigue questionnaire and was not related to muscle fatigue. Perceived fatigue assessed using questionnaires and changes in performance, measured using gait analysis, appear to capture two different features of fatigue in multiple sclerosis. In most patients, neurologists can detect the obvious fatigue syndrome in patients with MS using "classic" instruments. However, in some patients, the diagnosis of the fatigue syndrome is less definite, requiring a more comprehensive analysis. The FKS may be particularly useful for diagnosing fatigue in MS patients with comorbidity such as depression, sleep disturbances, low cardiovascular fitness and for evaluating intervention programs.

\section{Study limitations}

The main limiting factor in this study was that only one neurologist performed the classical fatigue assessment. Future studies should consider simultaneous assessments by many specialists. The physiotherapists should be given a slightly different task. Instead of rating the sequence in which the walking tests were performed, they should answer the question: "In which subject do you spot motor fatigue and in which do you not". In our study, we focused on patients with an EDSS between 1 and 6.5 (3 indicating the border between light and moderate disability) who were able to walk on a treadmill. Hence, the applicability and validity of our method using the FKS in other groups of MS patients is unknown, particularly for those with a more severely compromised walking distance. For instance, we did not assess the quality of sleep using the Epworth Sleepiness Scale, which might be relevant for assessing fatigue in patients with MS.

Acknowledgments The study was supported by the Lurija Institute, Kliniken Schmieder, Germany.
Conflicts of interest The authors declare no conflicts of interest with respect to the authorship and/or publication of this article.

Ethical standard The study was approved by the local ethics committee and conducted in accordance with the Declaration of Helsinki. All participants provided written informed consent prior to their participation.

\section{References}

1. Thickbroom GW, Sacco P, Faulkner DL, Kermode AG, Mastaglia FL (2008) Enhanced corticomotor excitability with dynamic fatiguing exercise of the lower limb in multiple sclerosis. J Neurol 255:1001-1005

2. Romani A, Bergamaschi R, Candeloro E, Alfonsi E, Callieco R, Cosi V (2004) Fatigue in multiple sclerosis: multidimensional assessment and response to symptomatic treatment. Mult Scler $10: 462-468$

3. Kluger BM, Krupp LB, Enoka RM (2013) Fatigue and fatigability in neurologic illnesses: proposal for a unified taxonomy. Neurology 80:409-416

4. Liepert J, Mingers D, Heesen C, Baumer T, Weiller C (2005) Motor cortex excitability and fatigue in multiple sclerosis: a transcranial magnetic stimulation study. Mult Scler 11:316-321

5. Bigland-Ritchie B, Rice CL, Garland SJ, Walsh ML (1995) Taskdependent factors in fatigue of human voluntary contractions. Adv Exp Med Biol 384:361-380

6. Petajan JH, White AT (2000) Motor-evoked potentials in response to fatiguing grip exercise in multiple sclerosis patients. Clin Neurophysiol 111:2188-2195

7. McDonald WI, Compston A, Edan G et al (2001) Recommended diagnostic criteria for multiple sclerosis: guidelines from the International Panel on the diagnosis of multiple sclerosis. Ann Neurol 50:121-127

8. Roelcke U, Kappos L, Lechner-Scott J et al (1997) Reduced glucose metabolism in the frontal cortex and basal ganglia of multiple sclerosis patients with fatigue: a $18 \mathrm{~F}$-fluorodeoxyglucose positron emission tomography study. Neurology 48:1566-1571

9. Krupp LB, LaRocca NG, Muir-Nash J, Steinberg AD (1989) The fatigue severity scale. Application to patients with multiple sclerosis and systemic lupus erythematosus. Arch Neurol 46:1121-1123

10. Schwartz JE, Jandorf L, Krupp LB (1993) The measurement of fatigue: a new instrument. J Psychosom Res 37:753-762

11. Fisk JD, Pontefract A, Ritvo PG, Archibald CJ, Murray TJ (1994) The impact of fatigue on patients with multiple sclerosis. Can J Neurol Sci 21:9-14

12. Guidelines MSCfCP (1998) Fatigue and multiple sclerosis. Paralyzed Veterans Association, Washington, DC

13. Penner IK, Raselli C, Stocklin M, Opwis K, Kappos L, Calabrese $\mathrm{P}$ (2009) The fatigue scale for motor and cognitive functions (FSMC): validation of a new instrument to assess multiple sclerosis-related fatigue. Mult Scler 15:1509-1517

14. Flachenecker P, Muller G, Konig H, Meissner H, Toyka KV, Rieckmann P (2006) "Fatigue" in multiple sclerosis. Development and validation of the "Wurzburger Fatigue Inventory for MS". Der Nervenarzt 77:165-166 (168-170, 172-164)

15. Sehle A, Mündermann A, Starrost K et al (2011) Objective assessment of motor fatigue in multiple sclerosis using kinematic gait analysis: a pilot study. J Neuroeng Rehabil 8:59

16. Harris GF, Smith PA (1996) Human motion analysis: current applications and future directions. Institute of Electrical and Electronics Engineers Press, New York 
17. Schablowski-Trautmann M, Gerner HJ (2006) State-space analysis of joint angle kinematics in normal treadmill walking. Biomed Eng 5:294-298

18. Rosenstein MT, Collins JJ, De Luca CJ (1993) A practical method for calculating largest Lyapunov exponents from small data sets. Phys D 65:117-134

19. Kantz H (1994) A robust method to estimate the maximal Lyapunov exponent of a time series. Phys Lett A 185:77-87

20. van Schooten KS, Rispens SM, Pijnappels M, Daffertshofer A, van Dieen JH (2013) Assessing gait stability: the influence of state space reconstruction on inter- and intra-day reliability of local dynamic stability during over-ground walking. J Biomech 46:137-141

21. Vieten MM, Sehle A, Jensen RL (2013) A novel approach to quantify time series differences of gait data using attractor attributes. PLoS ONE. doi:10.71371/journal.pone.0071824

22. Borg GA (1982) Psychophysical bases of perceived exertion. Med Sci Sports Exerc 14:377-381

23. Vieten MM (2004) Triple $F\left(F^{3}\right)$ Filtering of kinematic data. In: Proceeding: 22 International Symposium on Biomechanics in Sports, pp 446-449

24. Kurtzke JF (1983) Rating neurologic impairment in multiple sclerosis: an expanded disability status scale (EDSS). Neurology 33:1444-1452

25. Kuhner C, Burger C, Keller F, Hautzinger M (2007) Reliability and validity of the revised beck depression inventory (BDI-II). Results from German samples. Der Nervenarzt 78:651-656

26. Hautzinger M, Keller F, Kühner C (2008) Beck depressionsinventar (BDI-II), revision. Reportpsychologie 6:301-302

27. ATS Committee on Proficiency Standards for Clinical Pulmonary Function Laboratories (2002) ATS statement: guidelines for the six-minute walk test. Am J Respir Crit Care Med 166:111-117

28. Paul L, Enright MD (2003) The six-minute walk test. Respir Care 48:783-785

29. Braley TJ, Chervin RD, Segal BM (2012) Fatigue, tiredness, lack of energy, and sleepiness in multiple sclerosis patients referred for clinical polysomnography. Mult Scler. doi:10.1155/2012/ 673936
30. Labuz-Roszak B, Kubicka-Baczyk K, Pierzchala K, MachowskaMajchrzak A, Skrzypek M (2012) Fatigue and its association with sleep disorders, depressive symptoms and anxiety in patients with multiple sclerosis. Neurol Neurochir Pol 46:309-317

31. Kelleher KJ, Spence W, Solomonidis S, Apatsidis D (2010) The characterisation of gait patterns of people with multiple sclerosis. Disabil Rehabil 32:1242-1250

32. Spain RI, St George RJ, Salarian A et al (2012) Body-worn motion sensors detect balance and gait deficits in people with multiple sclerosis who have normal walking speed. Gait Posture 35:573-578

33. Sosnoff JJ, Sandroff BM, Motl RW (2012) Quantifying gait abnormalities in persons with multiple sclerosis with minimal disability. Gait Posture 36:154-156

34. Morris ME, Cantwell C, Vowels L, Dodd K (2002) Changes in gait and fatigue from morning to afternoon in people with multiple sclerosis. J Neurol Neurosurg Psychiatry 72:361-365

35. Remelius JG, Jones SL, House JD et al (2012) Gait impairments in persons with multiple sclerosis across preferred and fixed walking speeds. Arch Phys Med Rehabil 93:1637-1642

36. Geisler MW, Sliwinski M, Coyle PK, Masur DM, Doscher C, Krupp LB (1996) The effects of amantadine and pemoline on cognitive functioning in multiple sclerosis. Arch Neurol 53:185-188

37. Schwartz CE, Coulthard-Morris L, Zeng Q (1996) Psychosocial correlates of fatigue in multiple sclerosis. Arch Phys Med Rehabil 77:165-170

38. Krupp LB (2003) Fatigue in multiple sclerosis: definition, pathophysiology and treatment. CNS Drugs 17:225-234

39. Genova HM, Rajagopalan V, Deluca J et al (2013) Examination of cognitive fatigue in multiple sclerosis using functional magnetic resonance imaging and diffusion tensor imaging. PLoS ONE. doi:10.1371/journal.pone.0078811

40. Sharma KR, Kent-Braun J, Mynhier MA, Weiner MW, Miller RG (1995) Evidence of an abnormal intramuscular component of fatigue in multiple sclerosis. Muscle Nerve 18:1403-1411 